\title{
CLASH OF CULTURES. FORMEN DER BEGEGNUNG MIT DEM FREMDEN IN TEXTEN VON CHRISTIANE NEUDECKER
}

Agnes Bidmon ${ }^{1}$

Zusammenfassung: Vor dem Hintergrund der in einer globalisierten Welt immer drängenderen Frage nach einer adäquaten Verfahrensweise mit dem Fremden, das in einen hermeneutischen Verstehensprozess des Subjekts integriert werden muss, möglichst ohne eine gewaltsame sprachliche Deutungshoheit über das bzw. den Fremden auszuüben, untersucht der Beitrag zwei literarische Verfahrensweisen mit dem Fremden, die sich in den Texten der deutschen Gegenwartsautorin Christiane Neudecker finden. Es handelt sich dabei um das kulturwissenschaftliche Konzept des ,alter', d.h. um einen grundsätzlich verstehbaren, wenn auch nicht demselben kulturellen Horizont angehörigen Anderen einerseits, und um das Konzept des ,alius', d.h. ein dem menschlichen Verstehen und jeglicher Rationalität völlig entzogenes Anderes, andererseits.

Schlüsselwörter: Hermeneutik der Fremdheit, alter, alius, Interkulturalität, das Unheimliche

\begin{abstract}
The question of how a subject can understand the 'other', either a human or a non-human 'other', becomes more and more urgent in a globalized world as ours. It brings up the question, how we can encounter the 'other' without using a form of linguistic violence through putting him, her or it into our own patterns of understanding. The essay analyzes two cultural studies-based modes of dealing with this otherness in two novels of Christiane Neudecker, a contemporary German author: Firstly the concept of the 'alter', which aims at generally understandable forms of otherness such as intercultural encounters, and secondly the
\end{abstract}

\footnotetext{
1 Promotion im Fachbereich Neuere deutsche Literaturwissenschaft. Wissenschaftliche Mitarbeiterin am Lehrstuhl für Neuere deutsche Literaturgeschichte (Prof. Dr. Christine Lubkoll) und Koordinatorin des Elitestudiengangs Ethik der Textkulturen am Standort Erlangen. agnes.bidmon@fau.de
} 
concept of the 'alius', which describes encounters with the absolutely other that is excluded from rational ways of understanding in general.

Keywords: Hermeneutics of otherness, alter, alius, interculturality, das Unheimliche

\section{Hinführung}

Konzepte von Fremdheit besitzen im zeitgenössischen abendländischen Diskurs und nicht zuletzt auch in der aktuellen Theoriebildung von Geistes- und Kulturwissenschaften besondere Virulenz, wie sich unter anderem an der Fülle aktueller Publikationen zu diesem Themenkomplex ablesen lässt. In Zeiten zunehmender Globalisierung und damit einhergehender räumlicher wie kultureller Entgrenzung besteht eine immer dringlichere Notwendigkeit der Reflexion darüber, wie im ,global village' mit kulturellen, politischen oder religiösen Alteritäten umgegangen werden kann, ohne den Anderen auf unzulässige Weise festzuschreiben, ihn egologisch zu vereinnahmen und das dahinter stehende eigene Denk- und Wertesystem somit implizit oder explizit zu superiorisieren. Denn für diese Verfahrensweise mit Alteritäten weiß die Geschichte aller Zeiten und Orte zahllose blutige Beispiele anzuführen, seien es die mittelalterlichen Kreuzzüge oder die Eroberungsfahrten der Kolonialmächte und die damit verbundene Unterwerfung indigener Völker. Dass es sich bei diesen zivilisatorischen Katastrophen allerdings nicht um ein rein historisches Phänomen handelt, sondern um eine Problematik, die in der Gegenwart nichts von ihrer Dramatik und Brisanz eingebüßt hat und eine Reflexion über adäquate Umgangsweisen mit der Fremdheit umso dringlicher macht, belegen aktuelle Entwicklungen wie die rund um den so genannten ,Islamischen Staat', dessen kriegerischen Aktivitäten, die sich durch das Ausrufen des Dschihad auf ein sich selbst superiorisierendes Weltbild stützen, sowohl im Nahen Osten als auch bei Terroranschlägen in der westlichen Welt bereits unzählige Menschen zum Opfer gefallen sind.

Das Phänomen der Fremdheit einerseits und die Auslotung der Möglichkeiten, wie dieser Fremdheit begegnet werden kann andererseits, berühren also den Kern hermeneutischer Verstehens- und Deutungsprozesse und formulieren die Frage nach der Möglichkeit des Verstehens des Anderen einerseits und die damit untrennbar verbundene Frage nach der Verortung des eigenen Selbst in der Welt andererseits auf 
eine existenzielle Weise, denn, wie der Kulturwissenschaftler Klaus Lösch konstatiert:

Wenn denn Verstehens- und Beschreibungsbemühungen in der Regel nur zur Konstruktion von Alteritäten führen, die viel über den Konstruierenden aussagen mögen, aber nichts Adäquates über die Fremden [...], stellt sich die Frage umso drängender, ob bei einem Verzicht auf epistemische Gewalt gegenüber dem Fremden, auf die Unterwerfung des Fremden unter das eigene Kategoriensystem, überhaupt eine Annäherung an das Fremde und eine Fremdrepräsentation möglich sind (LÖSCH ,2012, s. 26).

Vor dem Hintergrund dieser fast schon aporetisch anmutenden Situation möchte der folgende Beitrag in einem ersten Schritt untersuchen, welche Antwortmöglichkeiten auf diese ganz grundsätzliche Problematik einer gewaltfreien Annäherung und Beschreibungsmöglichkeit des Fremden die kulturwissenschaftliche Theoriebildung entwickelt hat. Hierfür werden zwei Konzeptionen vorgestellt, die von je unterschiedlichen Situierungen des Fremden in Relation zur Weltwahrnehmung und -anschauung des beschreibenden Subjekts ausgehen und daraus resultierend verschiedene Beschreibungsmöglichkeiten der Andersheit des Anderen bzw. Fremden ableiten. In einem zweiten Schritt wird dann analysiert, auf welche Art und Weise literarische Texte mit der Problematik des Fremdverstehens verfahren und ob die näher vorgestellten theoretischen Konzepte hier einen Widerhall finden. Hierfür werden zwei Texte der Gegenwartsautorin Christiane Neudecker analysiert, die prädestiniert für diese Untersuchung sind, da sie beide nicht nur rein geographisch betrachtet in fernen Räumen angesiedelt sind und an fremden Orten spielen - zum einen in der (zur Entstehungszeit des Romans noch existenten) Militärdiktatur Burmas, zum anderen im undurchdringlichen Urwald Brasiliens -, sondern darüber hinaus auch je unterschiedliche Facetten von Fremdheit beleuchten und inszenieren. 


\section{Theorien der Fremdheit}

Die Unterscheidung zwischen dem Eigenen und dem Fremden ist ein menschliches Grundbedürfnis und kann daher als anthropologische Konstante verstanden werden, ,ist sie doch unabdingbare Voraussetzung für die Konstruktion eines Selbstbildes und Selbstverständnisses, mit einem Wort: der Identität" (LÖSCH, 2012, s. 26). Identität kann - und dies ist mittlerweile ein kulturwissenschaftlicher Gemeinplatz demzufolge nur relational in einer Form der Abgrenzung von einer Alterität entworfen werden. Von entscheidender Bedeutung ist dabei nun allerdings, auf welche Art und Weise mit dieser Alterität innerhalb des eigenen Beschreibungssystems verfahren wird. Daher unterscheidet die Forschung hier ganz grundsätzlich zwischen den beiden grundlegenden Konzepten des ,alter' und des ,alius', deren semantischer Gehalt innerhalb der Theoriebildung auf der jeweiligen lateinischen Bedeutung der Begriffe basiert:

Das lateinische alienus drückt im Unterschied zu peregrinus (ausländisch), externus (auswärtig) und barbarus (nicht römisch) die fremde Zugehörigkeit aus. [...] Das lateinische alter [...] bezeichnet den anderen von zweien im Unterschied zum einen ohne markierte differente Zugehörigkeit. So ist der andere als alter ego ein ego wie ich, nur eben anders, das heißt dasselbe in einer Varietät (TURK, 1990, s. 10).

,Alienus/alius', also der oder - im Deutschen - auch das Fremde, ist somit jenseits der dem ego bzw. dem Subjekt zur Verfügung stehenden Beschreibungskategorien angesiedelt, da ,alienus'-Phänomene nicht an der Welt des ego partizipieren und somit von dessen Begriffsinstrumentarium auch nicht erfasst werden können. Dies kann anhand der lateinischen Terminologie nochmals verdeutlicht werden: Schließlich bedeutet ,alienus` ursprünglich weder ,nicht-römisch ' noch ,ausländisch" und verweist somit auf eine Kategorie außerhalb der be- und erkannten und dadurch benennbaren Welt. ${ }^{2}$ Aufgrund dessen läuft das

2 „Während der Andere (Alter) als Gegenstück zur Selbstbestimmung in die bestehende Weltanschauung des Betrachters und seiner Gruppe integriert ist und oft stereotypen Charakter hat, ist der Fremde (Alius) unbestimmt und fern, befindet sich außerhalb der Welt des Betrachters und seiner Gruppe und stellt die 
diskursive Inventar des ego zwangsläufig ins Leere, wenn ,alienus'Phänomene begrifflich adäquat erfasst werden sollen, wodurch der bzw. das Fremde in dieser Unbeschreibbarkeit letztlich inkommensurabel und dadurch angsteinflößend bleiben, da sie nicht durch Benennung depotenziert werden können. Schließlich kann - wenn man die Mythostheorie des Philosophen Hans Blumenberg zugrunde legt - nur dasjenige Unbekannte innerhalb der menschlichen Lebenswelt in seiner Bedrohung gebannt werden, das vom Menschen auch benannt werden kann:

Angst [muß] immer wieder zur Furcht rationalisiert werden [...], sowohl in der Geschichte der Menschheit wie in der des Einzelnen. Das geschieht primär nicht durch Erfahrung und Erkenntnis, sondern durch Kunstgriffe, wie den der Supposition des Vertrauten für das Unvertraute, der Erklärungen für das Unerklärliche, der Benennungen für das Unbenennbare. Es wird eine Sache vorgeschoben, um das Ungegenwärtige zum Gegenstand der abwehrenden, beschwörenden, erweichenden oder depotenzierenden Handlung zu machen. Durch Namen wird die Identität solcher Faktoren belegt und angehbar gemacht, ein Äquivalent des Umgangs erzeugt. Was durch Namen identifizierbar geworden ist, wird aus seiner Unvertrautheit durch die Metapher herausgehoben, durch das Erzählen von Geschichten erschlossen in dem, was es mit ihm auf sich hat (BLUMENBERG, 1979, s. 11).

Insofern übernehmen Figuren des ,alius' im Kontrast zu Figuren des ,alter' letztlich auch gerade keine identitätskonstituierende bzw. stabilisierende Funktion, sondern führen im Gegenteil zu einer existenziellen Verunsicherung des Subjekts und seiner Weltwahrnehmung, da sie sich den dem Subjekt zugänglichen Kategorien entziehen, „mit denen wir die Welt strukturieren, ihr eine Ordnung aufzuerlegen versuchen, die eine Zone der Vertrautheit von der des

Identität und Gruppenideologie auf subversive Weise in Frage. Alius-Figuren haben keinen stereotypen, sondern utopisch-symbolischen bzw. mythischen Charakter. (EGGER, 2009, s. 14.) 
Unvertrauten scheidet" (LÖSCH, 2012, s. 26). ,Alius'-Phänomene gehen somit mit der Subversion von bestehenden (Denk-)Strukturen sowie dem Verlust von Orientierung und Weltvertrauen einher.

Phänomene des ,alter' hingegen erfassen das Gegenüber zwar als verschieden vom ego, also dem Subjekt, aber doch als grundsätzlich strukturell gleich, als gleichartig und gleichursprünglich ${ }^{3}$, wie auch hier bereits die lateinische Begriffsbedeutung belegt: ,alter' als „anderer von zweien" bildet somit den Teil einer bekannten Menge innerhalb der Welt des ego ab und ist dadurch auch mit den dem Subjekt zur Verfügung stehenden Begriffen beschreibbar. Die Betonung bei Figuren des ,alter liegt daher auf der Art und Weise des In-Bezug-Setzens von Subjekt und Anderem, woraus sich pejorative ebenso wie affirmierende bzw. superiorisierende Zuschreibungen ergeben können. Schließlich besteht nicht nur die - oben bereits kurz angeführte - Möglichkeit eines inferiorisierenden Alteritätskonstrukts, um sich der eigenen (individuellen wie kollektiven) Identität $z u$ versichern und diese zu bestärken, sondern auch die Möglichkeit der Xenophilie, die zu einer unreflektierten Aufwertung der Alterität im Verhältnis zur eigenen (individuellen wie kollektiven) Identität führt. Man denke hier nur an historische Phänomene wie den abendländischen Exotismus um 1800, der letztlich ebensolche Stereotypisierungen zur Folge hat wie die Xenophobie.

In der neuesten kulturwissenschaftlichen Theoriebildung wurde diese binäre - und letztlich statische - Opposition von ,alius' und ,alter mittlerweile jedoch aufgebrochen. Inzwischen steht nicht mehr eine Dichotomisierung, sondern vielmehr die Synthese der Begrifflichkeiten im Zentrum, um sich dem ganz Anderen, dem Fremden, möglichst adäquat sprachlich anzunähern und ihn/es beschreiben zu können, ohne epistemische Gewalt auszuüben und Festschreibungen vorzunehmen. Hierfür dienen unter anderem die Konzepte des litauisch-französischjüdischen Denkers Emmanuel Lévinas als Folie, der am Poststrukturalismus ebenso wie an der Phänomenologie geschult wurde und der die Begegnung mit jedem anderen Menschen sui generis als Verschränkung von ,alius' und ,alter ${ }^{6}$ auffasst. ${ }^{4}$ So konstatiert er in einem

\footnotetext{
${ }^{3}$ SCHLIEBEN-LANGE, 1998, s. 5.

${ }^{4}$ An dieser Stelle soll allerdings darauf hingewiesen werden, dass Lévinas selbst dem jüdischen Denkhorizont entstammt und somit nicht mit den Begriffen ,alter
} 
mittlerweile prominenten Diktum: „Einem Menschen begegnen heißt, von einem Rätsel wachgehalten zu werden“ (LÉVINAS, 2007). Das bedeutet, dass jedem Menschen - so vertraut er einem auch erscheinen mag - stets ein Kern der irreduziblen Fremdheit innewohnt, der jedes Subjekt einem anderen in seiner individuellen Subjekthaftigkeit unverfügbar macht und ihn daher vor einer endgültigen Festschreibung durch den Anderen bewahrt. Ein Gedanke, den auch der Philosoph Bernhard Waldenfels in seiner einschlägigen Studie über den Stachel des Fremden (WALDENFELS, 1990) aufgreift. Ziel bzw. Sinn der Begegnung ist daher auch nicht die letztliche Ergründung und Deutung des Anderen, die einer Gewaltausübung über den Anderen durch Unterwerfung in das eigene Kategoriensystem gleichkäme, sondern vielmehr die ethische Maxime der Anerkennung einer grundsätzlich vorhandenen Unergründbarkeit des Anderen - und nicht zuletzt des eigenen Selbst. ${ }^{5}$

\section{Konstruktionen von Fremdheit in der Literatur}

Im Folgenden soll nun untersucht werden, ob und wenn ja inwiefern die Texte von Christiane Neudecker mit den beiden Kategorien von ,alter' und ,alius' sowie deren Dynamisierung in der aktuellen Theoriebildung gelesen werden können bzw. wie literarische Texte mit der Inszenierung von Fremdheit verfahren.

\subsection{Die Autorin Christiane Neudecker}

Christiane Neudecker wurde 1974 in Nürnberg geboren und lebt mittlerweile in Berlin. Seit 2001 ist sie Mitglied des Künstlernetzwerks phase7performing.arts und inszenierte gemeinsam mit phase 7 zahllose und viel beachtete multimediale Performances. Ein besonderes Augenmerk von phase7 liegt dabei stets auf der innovativen Verschränkung von

und ,alius', die im abendländisch-ontologischen Denken verankert sind, operieren würde.

5 Vgl. hierzu auch Judith Butler: Kritik der ethischen Gewalt. AdornoVorlesungen 2002. Frankfurt a. M. 2003. Außerdem Stephanie Waldow: Schreiben als Begegnung mit dem Anderen. Zum Verhältnis von Ethik und Narration in philosophischen und literarischen Texten der Gegenwart. Paderborn 2013. 
traditionellen Kunstformen und neuen Technologien, wie sich u.a. an der 2013 erfolgten Inszenierung einer „Begehbaren Oper“ in der Deutschen Oper Berlin mit dem Titel Himmelsmechanik - Eine Entortung ablesen lässt. ${ }^{6}$ Die damit verbundene grundlegende Frage nach der Auslotung von Fakt und Fiktion sowie nach der Stellung des Menschen in einer medial und virtuell überformten Realität im 21. Jahrhundert durchzieht auch Neudeckers Erzählwerk. Mittlerweile hat Christiane Neudecker mehrere Erzählbände und Romane vorgelegt und wurde für ihre Art des Erzählens unter anderem von der Feuilleton-Redaktion der FAZ als „Meisterin der Atmosphäre" (HOFFMANN, 2008) bezeichnet. Für die folgenden Überlegungen werden Neudeckers Debut-Roman Nirgendwo sonst (2008) sowie die Titelerzählung ihres gleichnamigen Erzählbandes Das siamesische Klavier (2010) im Mittelpunkt der Betrachtung stehen.

\subsection{Die Begegnung mit der Fremde: ,Nirgendwo sonst ${ }^{\star}$}

Der Roman Nirgendwo sonst erzählt mithilfe zweier ineinander verwobener Erzählstränge von der Identitätssuche eines namenlosen Protagonisten, die sich in der kulturellen Fremde Burmas vollzieht. Wie fremd diese kulturelle Fremde ist, verdeutlicht der Text gleich zu Beginn, wenn die Rahmenbedingungen einer Reise in dieses Land geschildert werden:

Mit der einen Hand schält er das klebrige Laken von seinem Körper. Hält sich mit der anderen den Kopf. Dieses Pochen hinter den Schläfen. Der Whiskey, vielleicht. Dessen Namen er nicht kennt. Kein Jim Beam, Jack Daniels, erst recht kein Lagavulin. Keine Aufteilung in Bourbon, Single Malt, Double Wood. Keine westlichen Marken im sozialistischen Sperrgebiet. Du fährst, hatten die Freunde gesagt, in eines der einzigen drei Länder der Welt, in dem kein Coca Cola hergestellt wird. Wusstest du das. Wie fremd muss das sein (NEUDECKER, 2008, s. 14).

${ }^{6}$ Vgl. dazu URL: [http://www.schauinsblau.de/5-individuum-und-kosmos/bildand-ton/bildende-kunst/dass-die-welt-verrueckt-sein-mag/] (letzter Aufruf am 27.06.2016) 
Die Alterität im Sinne der äußersten vorstellbaren kulturellen Fremdheit stellt in diesem Text also eine geradezu paradigmatische Folie dar, um eine eigene Identität konstituieren zu können - allerdings nicht im Sinne der Konstruktion einer kollektiven Nationalidentität (deutsch burmesisch), sondern vielmehr eines individuellen Selbst. Der Protagonist in Neudeckers Text führt vor, dass man sich ganz der kulturellen Fremde aussetzen und sich in ihr verlieren muss, um neu zu sich finden $z \mathrm{u}$ können. Allerdings tritt mit der Touristin Ute, die dem Protagonisten während seiner Reise durch Burma immer wieder begegnet, auch eine weitere Zugangsweise zur Fremde auf, die gleichsam kontrapunktisch zu der des Protagonisten situiert ist. Denn Ute lässt sich im Verlauf der Reise nie wirklich auf das Fremde ein und begegnet ihm wirklich. Stattdessen behält sie stets einen touristischen Blick bei:

Doch Ute fragt nicht. Ihr Blick fliegt über die Ebene, sucht, so wirkt es, den nächsten Tempel, den sie besteigen könnte. Sie will sich von ihm nicht abbringen lassen. Will nicht hineingezogen werden in seine Gedanken. Sie ist hier, um die Bauten zu erkunden. Das hat sie ihm erzählt. Tempelräume will sie begehen, Wandmalereien in Höhlentempeln besuchen, glasierte Terrakotta-Tafeln betrachten, verwitternde Fresken, Ordinationshallen, abgestumpfte Buddhastatuen. Sie muss sich ablenken von der Arbeit daheim. Jede Jahr macht sie das: sie sucht den Abstand zu den Fällen, die sie im Frauenhaus betreut. Die räumliche Entfernung zu ihrem Arbeitsplatz ist dabei unerlässlich. Sie war schon in Laos, Vietnam, Kambodscha. Anders, hat sie erklärt, ließe sich ihr Beruf nicht ertragen (NEUDECKER, 2008. s. 199).

Die Fremde dient hier also als exotischer Gegenentwurf, um den belastenden heimischen Alltag für eine klar begrenzte Zeit hinter sich lassen zu können und andere Lebens- und Sichtweisen zu erkunden. Wie für viele Touristen besteht der Reiz der Fremde für die Figur Ute hauptsächlich darin, die Andersartigkeit des Reiselandes passiv zu rezipieren, um eigene Probleme für die Dauer der Reise überschreiben zu lassen. Auf der Folie des ,alter'-Konzeptes könnte man diese Haltung deshalb auch so beschreiben: Man reist, um gerade die positiven 
Differenzen zum Eigenen im Sinne einer Andersartigkeit bei grundlegender anthropologischer Gleichheit zu erfahren. Die negativen Gemeinsamkeiten, etwa die Erfahrungswerte gesellschaftlicher oder soziopolitischer Missstände, werden zwar momenthaft bemerkt, müssen aber in den Hintergrund treten bzw. haben keinen wirklichen Austausch zur Folge. Eine tatsächliche interkulturelle Begegnung ist demnach obsolet und muss scheitern, wie die Szene der Begegnung bzw. gerade der Nicht-Begegnung zwischen Touristen und burmesischen Frauen auf einem Touristenschiff exemplarisch belegt:

Einmal hält das Schiff. Schwenkt von der Mitte des Flusses auf eine lehmige Bucht zu. An der schmierigen Erdkante haben sich Einheimische aufgereiht, Frauen in bunten Gewändern, die den Passagieren Gegenstände entgegenstrecken: bestickte Decken, geflochtene Hüte, Bananen. Dollarscheine lassen die Italiener auf sie herabregnen, lassen Stoffbahn um Stoffbahn in das Innere des Schiffes laden. Eine Burmesin versucht, Ute [...] einen gewebten Longyi anzupreisen. Wie eine Signalfahne schwingt sie den rostbraunen Rock über ihrem Kopf, drückt die Knie durch, spannt die Waden, die Sprunggelenke, schiebt sich auf die Fußballen. Fünf Dollar will sie für den Rock, aber Ute schüttelt den Kopf. Die Frau lässt nicht locker, sie bleibt an Ute, Ute ist ihr Ziel. Und während das Schiff sich langsam abzuwenden beginnt, während die Drehzahl des Motors ansteigt, sich der Druck auf die Propellerwelle verstärkt, wird ihre Stimme schriller. Mit dem sich vergrößernden Abstand des Schiffes fallen die Zahlen, die ihr aus dem Mund fliehen, abwärts, vier, drei, zwei Dollar, bis sie plötzlich zum Sprung ansetzt und über den Lehmbruch in das schlammige Wasser springt. Kreischend watet sie dem Schiff hinterher, stemmt sich gegen den aufgewühlten Fluss, den Blick immer auf Ute geheftet, ein Dollar, ein Dollar. [...] „Perfum“, schreit die Frau zu Ute hinauf, als sie den Grund unter den Füßen zu verlieren beginnt, als sie rudern muss, um nicht unterzugehen, nicht Wasser zu schlucken, ,give me perfum". Und plötzlich greift Ute in die Tasche ihrer Hose, zieht ein Probefläschchen Eau de Cologne heraus, wickelt 
es in einen Dollarschein und wirft es über die Reling, der Frau in die aufgespannte, greifende Hand. Mit einem kräftigen Gegenwurf fliegt der Longyi in die Höhe, segelt über die Brüstung, verfolgt vom Grinsen der Soldaten [...].Ich konnte nicht anders, sagt sie und löst endlich ihren Blick vom sich entfernenden Ufer. Diese Frau da im Wasser, in dieser Dreckbrühe, und wir hier an Deck, auf diesem Luxusdampfer, dieser staatlichen Melkmaschine, was sollte ich tun. Dabei wollte ich den Rock gar nicht haben (NEUDECKER, 2008, s. 155).

Im Vordergrund steht für die Figur Ute im Verlauf der Reise also nicht die Begegnung mit den Menschen, sondern das Erlebnis exotischer landschaftlicher Schönheit sowie die ebenso pittoresken wie fremdartigen Bauwerke. Doch wird diese Faszination auch immer wieder gebrochen, da die Andersartigkeit Burmas nicht nur in kultureller und landschaftlicher Fremdheit besteht, sondern insbesondere im omnipräsenten und dadurch auch für Touristen spürbaren Klima eines totalitären Militärregimes:

Dass das Militär die Leute gezwungen habe, ihre eigenen Häuser abzureißen, sagt Ute jetzt. Wusstest du das. Sie ist in ihrem Kopf schon weitergelaufen, hat ihn und seine Antwort hier sitzen lassen, während sie selbst gedanklich durch das Tempelfeld streift. Die Freilegung der Tempel wurde von der Regierung befohlen, die Bewohner per Brief aufgefordert, Bagan zu verlassen. Ein ganzer Ort wurde verpflanzt, wurde verschoben, von Old Bagan nach New Bagan. Weil das Militär es so wollte. Das Schlimme daran ist, sagt Ute und deutet auf die Aussicht, dass das Ergebnis so schön ist. Schau dir das an (NEUDECKER, 2008. s. 200).

Während Utes Zugangsweise zur Fremde - sowohl räumlich als auch kulturell - demzufolge über „ein gesichertes Terrain der Eigenheit“ erfolgt und „sich auf eine bloße Fremdheit der Anderen [beschränkt]“, beginnt die Reise und die Begegnung mit der Fremde für den Protagonisten „im eigenen Haus der Fremdheit meiner selbst“ 
(WALDENFELS, 1995, s. 613). Diese Fremdheit des eigenen Selbst findet darin ihren Ausdruck, dass der Protagonist im Verlauf der Geschichte sich sukzessive von seiner bisherigen Identität und Lebensgeschichte, die in einigen Passagen des Textes immer wieder fragmentarisch eingeschoben ist und so vom Leser rekonstruiert werden kann, löst und sich ganz auf die Fremde einlassen muss, um neu zu sich $z \mathrm{u}$ finden und eine Identität konstituieren zu können. Auf diese Weise wird ein produktiver Umgang mit der Fremdheit ermöglicht, wie sich insbesondere anhand der Begegnung zwischen dem namenlosen Protagonisten und einem Einheimischen namens Mr. Khin in einem abgelegenen Bergdorf ablesen lässt. Und dies sogar in einer doppelten bzw. wechselseitigen Hinsicht: Zum einen setzt sich - wie erläutert - der Protagonist der kulturellen Fremde in Burma aus, zum anderen begegnet aber auch der einheimische Mr. Khin immer wieder Backpackern aus der westlichen Welt und somit dem für ihn Fremden. In Mr. Khin erhält die Fremdheit somit selbst eine eigene Stimme, die die unterschiedlichen Rollen der Touristen, also der Fremden aus burmesischer Perspektive, reflektiert:

Die Regierung unterschätze die Touristen. Nicht die organisierten Gruppen, die Bildungsreisenden, die habe der Staat tatsächlich im Griff. Aber die Backpacker, die Rucksacktouristen, sagt Mr. Khin, ihr seid ihnen gefährlich, denn ihr seid nicht steuerbar. Euch karrt man nicht von Pagode zu Pagode. Ihr kommt in Orte wie diesen. Nehmt Briefe, Bilder mit herein, heraus, erzählt allen die Wahrheit über uns, in Europa, Amerika, Australien. Bringt uns Informationen von jenseits der Grenzen. Darüber, wie man unser Land wahrnimmt. Über das, was wirklich passiert in der Welt. Ihr seid, sagt Mr. Khin, unsere trojanischen Pferde (NEUDECKER, 2008, s. 259).

Mr. Khins Perspektive lenkt den Blick darauf, dass in seiner Wahrnehmung das ,alter'-Konzept eine völlig andere Gewichtung als für Utes touristische Perspektive hat. Die Differenzen zwischen dem ihm Eigenen und dem davon Fremden werden dominiert von den Restriktionen im eigenen Land, in dem eine Militärdiktatur herrscht. Insofern ist die Begegnung mit den Fremden für Mr. Khin kein 
exotischer Gegenentwurf zur eigenen Lebenswelt, sondern vielmehr der existenzielle Versuch, buchstäblich in einen produktiven Austausch mit dem Anderen zu treten. Denn: Anders als Burmesen haben die Touristen aus der freien Welt beinahe unbegrenzten Zugang zu Informationen, verfügen über das Recht der freien Meinungsäußerung und können auf demokratischem Weg politischen Einfluss in ihren Heimatländern ausüben. Dieser interkulturelle Erfahrungsaustausch findet zunächst in Form eines Dialogs auf sprachlicher Ebene statt, indem Backpacker Mr. Khin Geschichten aus ihrer heimatlichen Erfahrungswelt erzählen. Diese individuellen Geschichten, die immer auch kollektive Geschichte repräsentieren, erscheinen in Khins Lebenswirklichkeit als Hoffnungsschimmer. So berichtete ihm einst eine aus Deutschland stammende Frau „von der Wiedervereinigung Deutschlands [...], der Demokratisierung des SED-Staats durch die friedlichen, gewaltfreien Demonstrationen. Dann die Maueröffnung: was für ein Symbol“ (NEUDECKER, 2008, s. 258). Daraus folgert Mr. Khin: „Eine Regierung lasse sich abschaffen, das habe der Niedergang der DDR gezeigt. Die Öffnung Burmas für den Tourismus sei da der Anfang."7 Indem Mr. Khin eine Analogie zwischen dem SED-Staat der DDR und der burmesischen Militärdiktatur herstellt, wird die negative Gemeinsamkeit der erlebten Unterdrückung im Gespräch mit dem Protagonisten in eine im positiven Sinne geteilte Möglichkeit der Veränderung gewendet, die das Eigene und das Fremde vor dem Hintergrund der für ihn fremden deutschen Geschichte miteinander verbindet und konkrete, beiderseitige Handlungsmöglichkeiten eröffnet:

Beim Abschied stehen sie lang in der halb offenen Hintertür. Einen Packen dünner Luftpostbriefe drückt ihm Mr. Khin in die Hand. Ob er die bitte einwerfen könne, wenn er außerhalb der Landesgrenze sei. Auf dem Heimflug, in Bangkok vielleicht, oder besser: in Eurem wiedervereinten Deutschland, das brächte mir Glück, ich bin sicher (NEUDECKER, 2008, s. 263).

\footnotetext{
${ }^{7}$ Christiane Neudecker: Nirgendwo sonst. München 2008. S. 258 f.

Dass dem tatsächlich so sein kann und die Perspektive von Mr. Khin folglich ex post betrachtet zukunftsweisend war, zeigt sich u.a. daran, dass die Militärdiktatur in Burma mittlerweile realpolitisch überwunden ist.
} 
Die Begegnung mit dem Fremden in Form der Wahrnehmung eines ,alter ' erscheint vor diesem Hintergrund demzufolge dann als produktiv, wenn sich ein unmittelbarer Austausch zwischen dem Eigenen und dem Fremden vollzieht, der sowohl das Fremde als auch das Eigene verändert daraus hervorgehen lässt.

In der Diktion des Philosophen Kurt Röttgers liegen den vorgestellten ,alter'-Zugangsweisen zum Fremden somit zwei metaphorisch benannte Modelle des Umgangs mit Fremderfahrung zugrunde: die Odyssee und der Exodus. ${ }^{8}$ Die Touristin Ute repräsentiert dabei das Modell der Odyssee, das eine Bereitwilligkeit zum buchstäblich umherirrenden Erfahren des Fremden kennzeichnet, die jedoch nicht am Verstehen, sondern an Bedürfnissen der Gewinnung von Sicherheiten für die eigene Wegbahnung in der Fremde ausgerichtet ist und getragen wird von dem Zutrauen, das Fremde zu bewältigen und anschließend heimzukehren. Dies belegt eine letzte zufällige Wiederbegegnung von Ute und dem Protagonisten am Flughafen: „Dass er jetzt viel besser aussehe als in Bagan, sagt sie ihm. Und wie sehr sie sich jetzt auf zu Hause freue, auf ihre Wohnung, ihr Bett, ihre Badewanne" (NEUDECKER, 2008, s. 269).

Der namenlose Protagonist hingegen setzt sich - wie bereits erwähnt - der Fremde mit seiner eigenen Identität nicht nur völlig aus, sondern gibt diese in der Begegnung mit dem Fremden sogar auf, wie die Szene des Abschieds nach dem letzten Gespräch mit Mr. Khin verdeutlicht:

Er stellt seinen Rucksack auf den Boden. Leise räumt er seine Sachen aus: die Kleider, den Wecker, die Medizin. Legt auch den Fotoapparat dazu, die Speicherkarte [...]. Behält nur ein T-Shirt zum Wechseln, seine Papiere, das Moskitonetz, die Reiseführer, ein paar Aspirin. Setzt sich schließlich tastend die Kontaktlinsen ein und legt seine Brille zuoberst auf den Stapel. [...] Als hätte das andere Ich, das er mit sich herumtrug, sich endlich abgestreift von ihm. Sähe ihm nach, ganz ruhig (NEUDECKER, 2008, s, 264).

${ }^{8}$ Vgl. hierzu Kurt Röttgers, Monika Schmitz-Emans: Philosophisch-literarische Reflexionen Bd. 9: Die Fremde. Essen 2007. 
Demzufolge praktiziert der Protagonist das Modell des Exodus, das eine Auslieferung an das Fremde bezeichnet, die ihn grundlegend verändert und ihn nicht mehr zum Ausgangspunkt zurückkehren lässt, und, dessen ist sich der Protagonist sicher: „Nur hier konnte geschehen, was mir geschehen ist. Nirgendwo sonst" (NEUDECKER, 2008. s. 260).

\subsection{Die Begegnung mit dem Fremden: ,Das Siamesische Klavier}

Auch im Erzählband Das Siamesische Klavier ist das Phänomen der Begegnung mit Fremdheit das vorherrschende Thema. Allerdings ist es dabei weniger die kulturelle Fremdheit, die im Mittelpunkt der Betrachtung steht, sondern vielmehr der Einbruch des Unfassbaren in die Lebenswirklichkeit der Figuren - und nicht zuletzt des Lesers. Besonders deutlich wird dies an der Titelgeschichte des Bandes, die vom mysteriösen Fund eines ungewöhnlichen Klaviers im brasilianischen Urwald handelt:

Wie ein riesiger Fehlwuchs beult sich auf der gegenüberliegenden Seite die zweite Klaviatur heraus. Richtig abartig ist das. Sie wissen auf einmal nicht mehr, wo vorne ist und wo hinten, plötzlich verlieren sie die Orientierung. [...] Es ist ein heimtückischer Januskopf, dieses Klavier, alles ist gedoppelt: das Spielwerk, die Mechanik, die Stimmwirbel, die Saiten, die Tastatur. Nur der verwachsene Korpus und der gemeinsame Resonanzboden halten es zusammen (NEUDECKER, 2010, s. 14).

Bereits in dieser Szene wird deutlich, dass die Begegnung mit dem Fremden in diesem Fall das gesamte Weltverständnis sowie die Weltwahrnehmung herausfordert und infrage stellt, da es sich um eine Konfrontation mit dem ganz Anderen handelt, das jenseits des menschlichen Erfahrungshorizontes liegt. Aus diesem Grund scheint es auch nahezu unmöglich, überhaupt Begrifflichkeiten für die Situation zu finden, die diese sprachlich fassbar machen könnten, weshalb bereits der Fund des Klaviers von der Erzählinstanz in mehreren Anläufen erzählt wird. Eine Verfahrensweise, die im Übrigen signifikanterweise einer mythischen Struktur entspricht, da die Funktion mythischen Erzählens ja gerade ist, Sachverhalte zu erzählen, die sich dem logisch-rationalen 
Zugriff entziehen und ein Verstehen verweigern. Dies geschieht insbesondere durch eine permanente Variation des narrativen Kerns, wodurch die vakant gebliebene Position von Antworten - also das, was nicht erzählt werden kann - weitergeschoben wird:

Irgendwer ist dann mit seiner Motorsäge zum Abholzen angerückt und der staunte nicht schlecht, als mit tiefem Stöhnen der Baum zur Erde sank und zwischen dem Krachen und Splittern der brechenden Äste und dem Kreischen aufflatternder Papageien plötzlich ein anderes, ein ganz anderes Geräusch zu hören war: ein dissonanter Akkord. [...] Aber so war es natürlich nicht. So einen Sturz hätte es ja niemals überlebt. Wobei: der tatsächliche Fundort klingt mindestens genauso erlogen. Wahrscheinlich basteln sie schon einen Mythos. Wer weiß, wo sie es wirklich herhaben, dieses merkwürdige Klavier. Man kann niemandem mehr trauen (NEUDECKER, 2010, s. 9f).

In der zweiten Version der Erzählung vom Fund des Klaviers hingegen wird es nicht bei Baumfällarbeiten, sondern stattdessen von einer deutschen Reisegruppe, die sich auf Expedition befindet, entdeckt:

[B]ei einer Urwaldexpedition, behaupten sie, kamen ein paar deutsche Touristen abhanden. Deren einheimischer Führer, irgendein glutäugiger Brasilianer namens Gonzales, hatte sich mit einer mitreisenden Dame mal eben kurz ins Farnkraut geschlagen - vorgeblich um ihr eine besonders seltene Orchideenart zu erörtern -, und als die beiden zurückkamen, war von den Teilnehmern der Expedition nur noch die Hälfte da. [...] Wie dem auch sei: da es langsam dämmerte und die ersten hungrigen Nachttiere im Waldgehölz zu rumoren begannen, schulterte einer der Teilnehmer, ein resoluter Schlachtermeister [...], den erschlafften Gonzales, griff sich dessen Machete und stapfte voran. Dass er keine Ahnung hatte, wo er hinging, zeigte sich, als die Gruppe wenig später vor einem irgendwie quadratisch aussehenden, aus dem Humusboden aufquellenden Hügel stand. Gerade wollte man zur 
Umrundung des Hindernisses ansetzen, als Gonzales plötzlich seinen Kopf vom Hintern des Schlachtermeisters hob und verwundert rufend auf ein paar Fenster deutete, die sich in dem grünen Hügelwirrwarr aus Lianenvorhang, Kletterefeu und Moosbewuchs abzeichneten (NEUDECKER, 2010, s. 10f.).

Die Gruppe erreicht eine verfallene Villa inmitten des Urwalds, in der sie neben dem Klavier auch dessen inzwischen längst verstorbenen Besitzer, den mürrischen Kautschukbaron Alvaro Luperce de Sanharó, dessen Tod aufgrund seines einsiedlerischen Lebenswandels von niemandem bemerkt worden war, auffinden:

Das Skelett des ehemaligen Barons jedenfalls lag auf einem der moskitonetzbespannten Betten im oberen Stock. Warum unsere Touristen da überhaupt hochgegangen sind, kann mir keiner zufriedenstellend erklären. Angeblich hat wieder der tapfere Schlachtermeister die Gruppe geführt. Er schlug vor, man solle, da nun auch der wieder munter gewordene Gonzales keine Ahnung mehr hatte, wo sie sich befanden, in der Villa nächtigen. Und müsse zu diesem Zweck das Gebäude erkunden (NEUDECKER, 2010, s. 12).

Neben dieser Art und Weise der inhaltlichen Darstellung sind es aber auch metanarrative Aspekte, mittels derer der Erzähler für ein zunehmendes Befremden beim Leser sorgt. Dass nämlich er selbst maßgeblichen Anteil an der Verunsicherung des Lesers hat, wird im Lauf der Geschichte immer deutlicher. Dies zeigt sich an den verdichteten Kommentaren, die im Lauf der Lektüre immer mehr Zweifel an der Zuverlässigkeit des Erzählers aufkommen lassen. So konstatiert er nicht zufällig: „Aber die Welt will betrogen sein“ (NEUDECKER, 2010, s. 20), um im weiteren Verlauf der Geschichte seine eigene Hybris offen zu legen: „Noch immer verströme ich eine Aura des Besonderen, eine Selbstbewusstheit, die immer noch Unerwartetes zulässt. Ja [...]. Ich kontrolliere alles" (NEUDECKER, 2010, s. 25). Schließlich kulminiert diese quasi-totalitäre Erzählsituation in direkten Leseransprachen, die das Unbehagen über das Vermittelte in Worte fassen und den Leser 
endgültig desorientieren: „Und auch Sie, ja, auch Sie haben jetzt endlich begriffen, dass hier etwas nicht stimmt" (NEUDECKER, 2010, s. 31).

Somit bildet die Erzählung Das Siamesische Klavier eine Begegnung mit der Fremdheit nicht nur ab, sondern ist für den Leser selbst eine Begegnung mit dem ganz Anderen, dem ,alius' in Form des Textes. Denn das Klavier verschmilzt am Ende der Geschichte zu einer Einheit mit dem Erzähler, der sich ebenso wie die Erzählung dadurch als selbst als janusköpfig erweist. Auf diese Art und Weise widersetzt sich das Ende der Erzählung einer Deutungshoheit durch den Rezipienten, ambivalente Lesarten werden ermöglicht und in der Schwebe gehalten, wodurch auch der hermeneutische Lektüreprozess unterwandert und letztlich eine Eindeutigkeit des Verstehens verweigert wird:

Und da weiß auch ich, was jetzt kommt, ich spüre es im Klavier, ich spüre das Gelächter in seinem Inneren, es kichert in sich hinein, während es mich spielen lässt, schneller und schneller [...]. Und ich versuche noch einmal, mich loszureißen, ich biege und winde mich auf dem Sitz herum, aber meine Füße kleben am Pedal, sie sind mit dem Klavier verwachsen, ich bin schon ein Teil von ihm, meine Finger hüpfen nicht mehr über die Tasten, sie lösen sich nicht, sind Verlängerungen der Spielmechanik geworden, wir betreten feuertrunken, Himmlische, dein Heiligthum! [...] und oben in der Königsloge sehe ich, wie da Silva den Kopf senkt, und ich fange an zu schreien, ich schreie und schreie, aber niemand hört mich, niemand kommt, es ist, als wäre ich gar nicht hier - Und, wissen Sie: vielleicht war ich das ja nie (NEUDECKER, 2010, s. 40).

\section{LITERATURVERZEICHNIS:}

BLUMENBERG, Hans Blumenberg. Arbeit am Mythos. Frankfurt a. M. 1979.

EGGER, Sabine Egger. Dialog mit dem Fremden. Erinnerung an den „europäischen Osten“ in der Lyrik Johannes Bobrowskis. Würzburg 2009.

HOFFMANN, Christina. Die verschwundene Dänin. Verfügbar in: http://www.faz.net/aktuell/feuilleton/buecher/rezensionen/belletrist 
ik/die-verschwundene-daenin-1671142.html. Letzter Aufruf am 08/07/2016.

KLAUS, Lösch. Das Fremde und seine Beschreibung. In: BRODRES, Simone; Gruß, Susanne; WALDOW, Stephanie (Hg.): Phänomene der Fremdheit - Fremdheit als Phänomen. Würzburg: 2012.

LÉVINAS, Emmanuel Lévinas. Die Spur des Anderen. Untersuchungen zur Phänomenologie und Sozialphilosophie. Freiburg i. Br./München 2007.

NEUDECKER, Christiane. Das siamesische Klavier. Unheimliche Geschichten. München 2010.

NEUDECKER, Christiane. Nirgendwo sonst. München 2008.

SCHLIEBEN-LANGE, Brigitte. Vorwort. In: Zeitschrift für Literaturwissenschaft und Linguistik 110: Alterität (28/1998).

TURK, Horst. Alienität und Alterität als Schlüsselbegriffe einer Kultursemantik. In: Jahrbuch für Internationale Germanistik 22 (1990).

WALDENFELS, Bernhar. Das Eigene und das Fremde. In: Deutsche Zeitschrift für Philosophie 43 (4/1995).

WALDENFELS, Bernhard. Der Stachel des Fremden. Frankfurt a. M. 1990.

Recebido em: 22 de agosto de 2016.

Aceito em : 10 de dezembro de 2016. 